\title{
Burkholderia thailandensis Isolated from Infected Wound, Arkansas, USA
}

\section{Jay E. Gee, Mindy G. Elrod, Christopher A. Gulvik, Dirk T. Haselow, Catherine Waters, Lindy Liu, Alex R. Hoffmaster}

The bacterium Burkholderia thailandensis, a member of the Burkholderia pseudomallei complex, is generally considered nonpathogenic; however, on rare occasions, $B$. thailandensis infections have been reported. We describe a clinical isolate of $B$. thailandensis, BtAR2017, recovered from a patient with an infected wound in Arkansas, USA, in 2017.

$B$ urkholderia thailandensis is a member of the Burkholderia pseudomallei complex and is generally considered nonpathogenic (1). B. pseudomallei causes the disease melioidosis and can be fatal even when properly treated (1-3). B. thailandensis was first discovered in Thailand and was differentiated from B. pseudomallei phenotypically by its ability to assimilate arabinose (3). B. thailandensis, like B. pseudomallei, naturally occurs in the environment (e.g., in moist soils) and is associated with tropical or subtropical climates $(3,4)$. Environmental isolates of $B$. thailandensis have been predominantly recovered in Southeast Asia, with sporadic reports in other regions such as in Australia, which is considered the ancestral origin for B. pseudomallei based on analysis of genomic single-nucleotide polymorphisms (SNPs) (4-6).

The phenotypic similarities are substantial among members of the B. pseudomallei complex, other Burkholderia species, and other bacteria such as Pseudomonas spp. Laboratory personnel unfamiliar with $B$. pseudomallei often have difficulty identifying it using microbiologic methods commonly available in clinical settings. Automated biochemical systems might also misidentify $B$. pseudomallei because of an insufficient number of reference strains in their databases. Incorrect identification can delay appropriate antimicrobial therapy $(1,4,7)$.

Although rare, B. thailandensis infections in humans have been reported. The first case reported in the literature occurred in or before 1999 in Thailand in a 16-year-old

Author affiliations: Centers for Disease Control and Prevention,

Atlanta, Georgia, USA (J.E. Gee, M.G. Elrod, C.A. Gulvik, L. Liu, A.R. Hoffmaster); Arkansas Department of Health, Little Rock, Arkansas, USA (D.T. Haselow, C. Waters)

DOI: https://doi.org/10.3201/eid2411.180821 boy who suffered compound fractures in a motorcycle accident (8). The second case occurred in 1997 in a 76-yearold man in Louisiana, USA, who had a pleural wound; this case resulted in recovery of isolate $\mathrm{H} 0587$ (also known as CDC2721121). Further details on the wound and whether it was acquired locally or whether the patient had a travel history to areas endemic for $B$. thailandensis are not available. The third case occurred in 2003 in a previously healthy 2-year-old boy in Texas, USA, who aspirated water from a ditch after a car accident; this case resulted in recovery of isolate TXDOH (also known as CDC3015869) (9). The fourth case occurred in 2011 in a 42-year-old man in Malaysia who had a foot abscess with ankle swelling and skin cellulitis (10). The fifth report was a fatal case that occurred in 2013 in a 67-year-old man who was treated at a hospital in Chongquing, China (11); however, subsequent correspondence suggested that the species had been identified incorrectly and was most likely B. pseudomallei (12).

In April 2017, a 29-year-old woman with diabetes in Arkansas, USA, crashed into a large metal trash bin while driving an all-terrain vehicle, resulting in an open bone forearm fracture. Treatment of the wound included installation of a metal plate. Approximately 3 months later, the patient returned to the hospital because of a 2-week history of swelling of her forearm. An isolate was recovered from pure culture graded $1+$ (trace growth) from a deep operative tissue specimen from the forearm wound. Initial antimicrobial treatment consisted of piperacillin/tazobactam, vancomycin, and cefazolin. Testing of the isolate using the Microscan Walkaway automated biochemical system (Beckman Coulter, Atlanta, GA, USA) by the hospital laboratory identified $B$. pseudomallei. Based on this result, the Arkansas Department of Health (DoH) contacted the Centers for Disease Control and Prevention (CDC) about a suspected case of melioidosis. After the hospital transferred the isolate to the Arkansas DoH, B. pseudomallei was ruled out by using biochemical and PCR testing. The isolate was presumptively identified as $B$. thailandensis by using the Bruker MALDI Biotyper mass spectrometer (Bruker Daltonics, Billerica, MA, USA). Arkansas DoH forwarded the isolate, designated BtAR2017, to CDC for confirmation and further characterization. The patient was discharged with 6 weeks of intravenous ceftazidime treatment. She last saw her healthcare provider in December 2017 and was reported completely healed. 


\section{The Study}

Biochemical testing, including arabinose assimilation, identified isolate BtAR2017 as B. thailandensis. We extracted DNA from the isolate for next-generation sequencing (online Technical Appendix, https://wwwnc.cdc.gov/ EID/article/24/11/18-0821-Techapp1.pdf). We analyzed the genome of BtAR2017 for multilocus sequence typing (MLST), which yielded sequence type (ST) 101. ST101 was previously identified in $B$. thailandensis isolates TXDOH and H0587 (9). Although MLST is commonly used to subtype members of the $B$. pseudomallei complex, it has only a moderate level of resolution and, because of recombination, might not reflect actual levels of relatedness $(5,13)$.

For high-resolution analysis, we compared the genome of BtAR2017 with a reference panel of publicly available $B$. thailandensis genomes (Table). A dendrogram based on the SNP analysis indicates that BtAR2017 clusters with $\mathrm{H} 0587$ and TXDOH (Figure). Also within the BtAR2017 cluster is E555, an environmental isolate recovered in Cambodia in 2005, and strain 2.1, which metadata in the National Center for Biotechnology Information entry indicate was recovered in June 2017 from soil in Vietnam (14). This cluster appears as an outlier subgroup compared with other examples of B. thailandensis, including E264 (type strain). MLST indicates that E555 is ST696, which is a single-locus variant of ST101. Comparison of the genome sequences indicates $>4,700$ core SNPs between BtAR2017 and E555, compared with $>32,700$ SNPs between BtAR2017 and E264' . BtAR2017 has $>2,200$ SNPs compared with H0587 but $>5,900$ SNPs compared with TXDOH.

Sim et al. (14) previously noted this outlier subgroup and postulated that its members, including E555, might be more virulent than typical examples of $B$. thailandensis. However, their studies challenging BALB/c mice and the nematode Caenorhabditis elegans with E555 did not reveal any significant difference in virulence compared with E264 ${ }^{\mathrm{T}}$ (14). Another study by Deshazer using TXDOH and H0587 to challenge mice and hamsters indicated that in these models TXDOH also had a similar level of virulence as E264 ${ }^{\mathrm{T}}$, whereas H0587 was avirulent (15). E555 is known to have features such as a $B$. pseudomallei-like capsular polysaccharide (14). We tested BtAR2017 for B. pseudomallei capsular polysaccharide, but results were negative.

We analyzed average nucleotide identity (ANI) and determined that the genome for BtAR2017 has $98.99 \%$ identity with that of $\mathrm{E} 264^{\mathrm{T}}$, which is above the $95 \%$ threshold commonly used for distinguishing species. The BtAR2017 genome also had an ANI of $99.75 \%$ compared with TXDOH but only $92.7 \%$ compared with K96243, a representative $B$. pseudomallei strain used in lieu of the species type strain. Although BtAR2017 is in the outlier subgroup resolved by genomic SNP analysis, these ANI results suggest that BtAR2017 and other members of the subgroup are $B$. thailandensis and not a novel species.

\section{Conclusions}

The recovery of the clinical isolates suggests that $B$. thailandensis might be endemic to the continental United States

\begin{tabular}{|c|c|c|c|c|c|c|c|}
\hline Isolate & Other identifiers & Origin & Source & STt & GenBank accession nos. & ANI & SNP \\
\hline 82172 & $34 ; 2002721621$ & France & Horse (foal) & 73 & NZ LNNG00000000 & & $\mathrm{X}$ \\
\hline Bt4 & 49639 & Australia & Environmental & 699 & $\mathrm{NZ}$ ABBH00000000 & & $\mathrm{X}$ \\
\hline E1 & & $\begin{array}{l}\text { Papua New } \\
\text { Guinea }\end{array}$ & Environmental & 669 & NZ_LOXF00000000 & & $\mathrm{X}$ \\
\hline E254 & & Thailand & Environmental & 345 & $\begin{array}{l}\text { NZ_CP004381.1; } \\
\text { NZ_CP004382.1 }\end{array}$ & & $X$ \\
\hline E264 & ATCC 700388 & Thailand & Environmental & 80 & СР008785.1; СР008786.1 & $\mathrm{X}$ & $\mathrm{X}$ \\
\hline E444 & & Thailand & Environmental & 79 & $\begin{array}{l}\text { NZ_CP004117.1; } \\
\text { NZ_CP004118.1 }\end{array}$ & & $x$ \\
\hline E555 & & Cambodia & Environmental & 696 & NZ_AECN00000000 & & $x$ \\
\hline H0587 & $\begin{array}{l}\text { BtCDC2721121; } \\
2002721121\end{array}$ & $\begin{array}{l}\text { USA } \\
\text { (Louisiana) }\end{array}$ & Human & 101 & $\begin{array}{l}\text { NZ__CP013409.1; } \\
\text { NZ_CP013410.1 }\end{array}$ & & $x$ \\
\hline K96243 & & Thailand & Human & 10 & $\begin{array}{l}\text { NC̄_006350.1; } \\
\text { NC_006351.1 }\end{array}$ & $X$ & \\
\hline MSMB59 & MSMB0059 & Australia & Environmental & 699 & $\begin{array}{l}\text { NZ_CP004385.1; } \\
\text { NZ_CP004386.1 }\end{array}$ & & $X$ \\
\hline MSMB60 & MSMB0060 & Australia & Environmental & 699 & NZ_LOXG00000000 & & $\mathrm{X}$ \\
\hline Phuket 4W-1 & & Thailand & Environmental & 80 & NZ_AQQJ01000000 & & $x$ \\
\hline Strain 2.1 & & Vietnam & Environmental & 696 & PHRD00000000 & & $x$ \\
\hline TXDOH & $\begin{array}{l}\text { CDC3015869; } \\
2003015869\end{array}$ & USA (Texas) & Human & 101 & $\begin{array}{l}\text { NZ_CP013360.1; } \\
\text { NZ_CP013361.1 }\end{array}$ & $X$ & $x$ \\
\hline $\begin{array}{l}\text { USAMRU Malaysia no. } \\
20\end{array}$ & $\begin{array}{l}\text { Malaysia \#20; } \\
2002721744\end{array}$ & Malaysia & Unknown & 80 & $\begin{array}{l}\text { NZ_CP004383.1; } \\
\text { NZ CP004384.1 }\end{array}$ & & $X$ \\
\hline
\end{tabular}




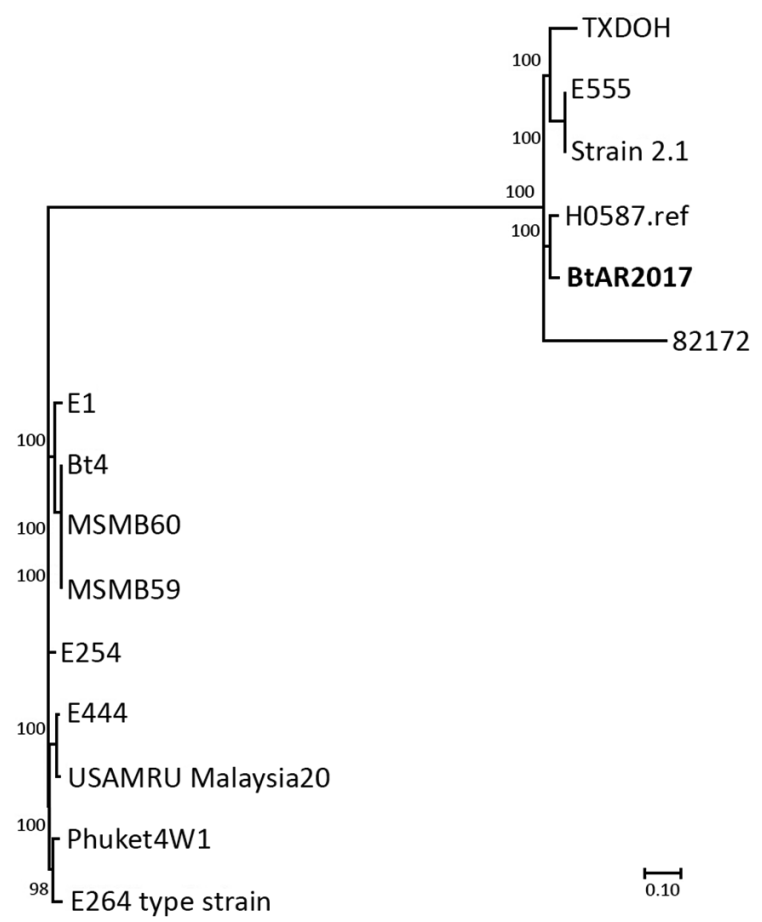

Figure. Dendrogram used for characterization of Burkholderia thailandensis isolate (bold) from an infected wound, Arkansas, USA, 2017; compared with reference isolates. Generated in MEGA 7.0 software (http://www.megasoftware.net) from results of maximum-parsimony phylogenetic analysis of core singlenucleotide polymorphisms from available $B$. thailandensis genomes (conducted by using Parsnp, a component of the Harvest 1.3 software suite [https://github.com/marbl/harvest]). Scale bar indicates number of substitutions per SNP.

and capable of causing opportunistic infections associated with traumatic injuries. Because of the limited number of reports, this bacterium's geographic range in North America is unknown. Two of the 3 clinical isolates recovered in the United States are now well documented and were acquired in the southern states of Texas and Arkansas, possibly reflecting the affinity for warm climates associated with most members of the $B$. pseudomallei complex (the exception being $B$. mallei, which is host-adapted to equids and does not persist in the environment). These 3 clinical isolates also are members of the outlier subgroup on the dendrogram (Figure), which suggests a genetic bottleneck or discrete seeding event for the United States, assuming the Louisiana case was acquired locally. Further study of $B$. thailandensis will improve our knowledge of its geographic range and ability to cause infections.

\section{Acknowledgments}

We thank the CDC Biotechnology Core Facility Branch (Division of Scientific Resources, National Center for Emerging and Zoonotic Infectious Diseases) for performing Illumina sequencing.
Our analysis made use of the Burkholderia pseudomallei MLST website (http://pubmlst.org/bpseudomallei) sited at the University of Oxford. The development of this site has been funded by the Wellcome Trust.

\section{About the Author}

Dr. Gee is a research biologist in the Bacterial Special Pathogens Branch, Division of High-Consequence Pathogens and Pathology, National Center for Emerging and Zoonotic Infectious Diseases, Centers for Disease Control and Prevention. His primary research interest is molecular epidemiology of members of the B. pseudomallei complex and other emerging bacterial pathogens.

\section{References}

1. Tuanyok A, Mayo M, Scholz H, Hall CM, Allender CJ, Kaestli M, et al. Burkholderia humptydooensis sp. nov., a new species related to Burkholderia thailandensis and the fifth member of the Burkholderia pseudomallei complex. Appl Environ Microbiol. 2017;83:e02802-16. http://dx.doi.org/10.1128/AEM.02802-16

2. Wiersinga WJ, Virk HS, Torres AG, Currie BJ, Peacock SJ, Dance DAB, et al. Melioidosis. Nat Rev Dis Primers. 2018;4:17107. http://dx.doi.org/10.1038/nrdp.2017.107

3. Brett PJ, DeShazer D, Woods DE. Burkholderia thailandensis sp. nov., a Burkholderia pseudomallei-like species. Int J Syst Bacteriol. 1998;48:317-20. http://dx.doi.org/10.1099/ 00207713-48-1-317

4. Levy A, Merritt AJ, Aravena-Roman M, Hodge MM, Inglis TJ. Expanded range of Burkholderia species in Australia. Am J Trop Med Hyg. 2008;78:599-604.

5. Pearson T, Giffard P, Beckstrom-Sternberg S, Auerbach R, Hornstra H, Tuanyok A, et al. Phylogeographic reconstruction of a bacterial species with high levels of lateral gene transfer. BMC Biol. 2009;7:78. http://dx.doi.org/10.1186/1741-7007-7-78

6. Ginther JL, Mayo M, Warrington SD, Kaestli M, Mullins T, Wagner DM, et al. Identification of Burkholderia pseudomallei near-neighbor species in the Northern Territory of Australia. PLoS Negl Trop Dis. 2015;9:e0003892. http://dx.doi.org/10.1371/ journal.pntd.0003892

7. Hoffmaster AR, AuCoin D, Baccam P, Baggett HC, Baird R, Bhengsri S, et al. Melioidosis diagnostic workshop, 2013. Emerg Infect Dis. 2015;21.

8. Lertpatanasuwan N, Sermsri K, Petkaseam A, Trakulsomboon S, Thamlikitkul V, Suputtamongkol Y. Arabinose-positive Burkholderia pseudomallei infection in humans: case report. Clin Infect Dis. 1999;28:927-8. http://dx.doi.org/10.1086/517253

9. Glass MB, Gee JE, Steigerwalt AG, Cavuoti D, Barton T, Hardy RD, et al. Pneumonia and septicemia caused by Burkholderia thailandensis in the United States. J Clin Microbiol. 2006;44:4601-4. http://dx.doi.org/10.1128/JCM.01585-06

10. Zueter AM, Abumarzouq M, Yusof MI, Wan Ismail WF, Harun A. Osteoarticular and soft-tissue melioidosis in Malaysia: clinical characteristics and molecular typing of the causative agent. J Infect Dev Ctries. 2017;11:28-33. http://dx.doi.org/10.3855/ jidc.7612

11. Chang K, Luo J, Xu H, Li M, Zhang F, Li J, et al. Human infection with Burkholderia thailandensis, China, 2013. Emerg Infect Dis. 2017;23:1416-8. http://dx.doi.org/10.3201/eid2308.170048

12. Dance DAB, Sarovich D, Price EP, Limmathurotsakul D, Currie BJ. Human Infection with Burkholderia thailandensis, China, 2013. Emerg Infect Dis. 2018;24:953-4. http://dx.doi.org/ 10.3201/eid2405.180238 
13. Gee JE, Gulvik CA, Elrod MG, Batra D, Rowe LA, Sheth M, et al. Phylogeography of Burkholderia pseudomallei isolates, Western Hemisphere. Emerg Infect Dis. 2017;23:1133-8. http://dx.doi.org/ 10.3201/eid2307.161978

14. Sim BM, Chantratita N, Ooi WF, Nandi T, Tewhey R, Wuthiekanun V, et al. Genomic acquisition of a capsular polysaccharide virulence cluster by non-pathogenic Burkholderia isolates. Genome Biol. 2010;11:R89. http://dx.doi.org/10.1186/ gb-2010-11-8-r89
15. DeShazer D. Virulence of clinical and environmental isolates of Burkholderia oklahomensis and Burkholderia thailandensis in hamsters and mice. FEMS Microbiol Lett. 2007;277:64-9. http://dx.doi.org/10.1111/j.1574-6968.2007.00946.x

Address for correspondence: Jay E. Gee, Centers for Disease Control and Prevention, 1600 Clifton Rd NE, Mailstop G34, Atlanta, GA 30329-4027, USA; email: xzg4@cdc.gov

\section{October 2017: Bacterial Infections}

- Fatal Rocky Mountain Spotted Fever along the United States-Mexico Border, 2013-2016

- Surveillance of Extrapulmonary Nontuberculous Mycobacteria Infections, Oregon, USA, 2007-2012

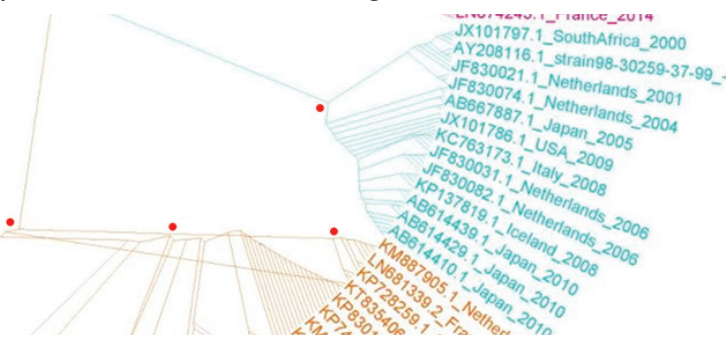

- Investigation of Outbreaks of Salmonella enterica Serovar Typhimurium and Its Monophasic Variants Using Whole-Genome Sequencing, Denmark

- Enteric Infections Circulating during Haij Seasons, 2011-2013

- Economic Assessment of Waterborne Outbreak of Cryptosporidiosis

- Antimicrobial Drug Prescription and Neisseria gonorrhoeae Susceptibility, United States, 2005-2013

- Poliovirus Excretion in Children with Primary Immunodeficiency Disorders, India

- Disease Burden of Clostridium difficile Infections in Adults, Hong Kong, China, 2006-2014

- Molecular Tracing to Find Source of Protracted Invasive Listeriosis Outbreak, Southern Germany, 2012-2016

- Dengue Virus 1 Outbreak in Buenos Aires, Argentina, 2016

- Mild Illness during Outbreak of Shiga Toxin-Producing Escherichia coli O157 Infections Associated with Agricultural Show, Australia

- Enterovirus D68-Associated Acute Flaccid Myelitis in Immunocompromised Woman, Italy

- Diagnosis of Fatal Human Case of St. Louis Encephalitis Virus Infection by Metagenomic Sequencing, California, 2016

- Usutu Virus RNA in Mosquitoes, Israel, 2014-2015
- Macrolide-Resistant Mycoplasma pneumoniae Infection, Japan, 2008-2015

- Epidemiology of Reemerging Scarlet Fever, Hong Kong, 2005-2015

- Off-Label Use of Bedaquiline in Children and Adolescents with Multidrug-Resistant Tuberculosis

- Monitoring Avian Influenza Viruses from Chicken Carcasses Sold at Markets, China, 2016

- Bedaquiline and Delamanid Combination Treatment of 5 Patients with Pulmonary Extensively Drug-Resistant Tuberculosis

- Hantavirus Pulmonary Syndrome Caused by Maripa Virus in French Guiana, 2008-2016

- Berlin Squirrelpox Virus, a New Poxvirus in Red Squirrels, Berlin, Germany

- Bedaquiline and Linezolid for Extensively Drug-Resistant Tuberculosis in Pregnant Woman

- Mycobacterium riyadhense in Saudi Arabia

- Carbapenemase VCC-1-Producing Vibrio cholerae in Coastal Waters of Germany

- Autochthonous Transmission of East/Central/South African Genotype Chikungunya Virus, Brazil

- Fluoroquinolone-Resistant Alcaligenes faecalis Related to Chronic Suppurative Otitis Media, Angola

- Spread of Chikungunya Virus East/Central/South African Genotype in Northeastern Brazil

- Familial Transmission of emm12 Group A Streptococcus

- Six-Month Response to Delamanid Treatment in MDR TB Patients

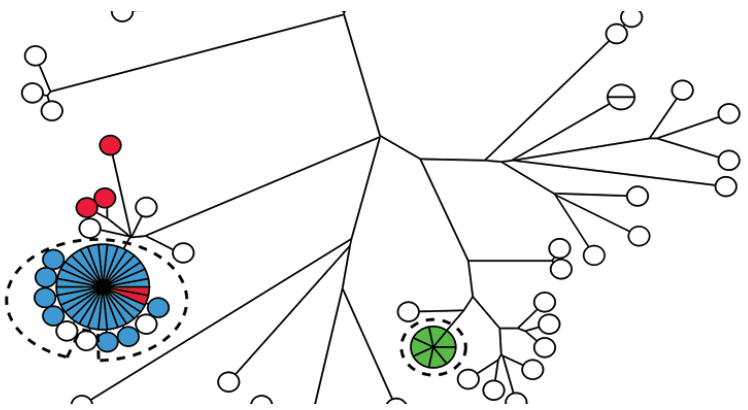

